\title{
HACIA UNA MODA SOSTENIBLE Y ECOLÓGICA
}

\author{
TOWARDS A SUSTAINABLE AND EGOLOGICAL FASHION
}

\begin{abstract}
Zeas Silvia
Muestra desde temprana edad su pasión por el diseño y el arte, lo que hace que realice sus estudios de Diseño Textil y Moda en la Universidad del Azuay, años después obtiene el título de Máster en Diseño Textil y Moda en Milano, Italia; y otro como Máster en Proyectos de Diseño en la Universidad del Azuay. Sus propuestas han ganado importantes premios, como por ejemplo: Mejor Traje Típico en diferentes eventos nacionales e internacionales, en su trayectoria ha participado en más de sesenta desfiles, tanto nacionales como internacionales. Entre sus reconocimientos se puede mencionar que fue invitada por el canal CNN en Español, ha exhibido sus diseños en la Casa Blanca de Estados Unidos y en la tienda de moda Macy's. Su trabajo con artesanos de diferentes comunidades la ha llevado a publicar sus experiencias en revistas académicas y a participar como ponente en la Bienal de Diseño en República Dominicana en 2016. Actualmente trabaja en la publicación de su tesis de maestría titulada "Entretejidos de Moda"; lleva a cabo un proyecto de investigación denominado "Registro icoográfico de las fajas del Cañar"; desarrolla un proyecto de vinculación con la comunidad de Tushin en Biblián y se desenvuelve como docente tiempo completo en la Carrera de Diseño Textil y Moda de la Universidad del Azuay.
\end{abstract}

silviazeas@uazuay.edu.ec

Fecha de recepción: 23 de febrero, 2017 / Aceptación: 31 de marzo, 2017. 


\section{Resumen}

La producción de textiles y modas a nivel industrial ha causado grandes problemas de contaminación ambiental, problema que ocasiona no solo la producción masiva, sino también la de menor escala y la producción de cada uno de los ciclos de vida de una prenda. El presente trabajo analiza los diferentes procesos que intervienen en la producción de una prenda y las posibilidades de solución eficiente y viable para disminuir la contaminación ambiental.

Palabras clave: industria textil, moda, moda ecológica, sustentabilidad.

\section{Abstract}

The production of textiles and fashions at industrial level has caused great environmental problems, which is not only caused by mass production, but also by smaller- scale project and the production of each of the life cycles of the garment. The present work analyzes the different processes involved in the production of a garment and the possibilities for an efficient and viable solution to reduce pollution.

Keywords: textile industry, fashion, ecological fashion, sustainability. 


\section{INTRODUCCIÓN}

La insaciable avidez por la renovación en la moda no solo está provocada por los imperios comerciales y el disfrute de los consumidores de lo "nuevo", sino también por la fascinación hacia la imagen y la narración.

Clarie Wilcox

El siguiente trabajo propone un modelo sustentable, en donde prime el comercio justo, la ética con los trabajadores y procesos sostenibles en todo el ciclo de producción; se establecen alternativas sostenibles en el ciclo de vida de una prenda que incluyan diferentes procesos, materiales, distribución y consumo de la indumentaria. La propuesta ecológica pretende vincular la academia con la industria y a las empresas públicas y privadas en programas interdisciplinarios. Además, analiza el impacto que ocasiona la moda en el planeta, en porcentajes reales, como efecto del consumo de la moda a nivel mundial y local. Se analizan dos cuadros descriptivos; uno con los impactos de los diferentes procesos que implica la moda y cómo repercuten en el medioambiente y otro, con las alternativas sostenibles y ecológicas que se podrían aplicar en cada una de las fases del ciclo de vida de una prenda. Toma como ejemplo de modelo sustentable a una empresa ecuatoriana que fabrica hilo reciclado de botellas elaboradas en plástico PET, hilo que es empleado en la producción de pantalones jean y bolsos.

\section{CONSUMO DE LA MODA}

Desde la Revolución Industrial, la producción en serie ha ganado terreno en desmedro de la producción artesanal y las de menor escala. Este fenómeno ha generado a su vez, grandes volúmenes de desechos, mayor requerimiento de personal y, en muchos casos, una producción desmedida, pero no se ha tomado en cuenta los daños colaterales que esto ocasiona. Nos hemos convertido en sociedades de consumo que habitamos en medio de industrias capitalistas.

En nuestra sociedad de consumo es fácil llegar a ser víctimas de lo que se considera "consumo compulsivo»: comprar por comprar, en lugar de hacerlo para satisfacer una necesidad o deseo. Ir de compras se ha convertido en una actividad de ocio, de manera que no concebimos que podamos dar un paseo sin comprar nada por el camino" (Ecologistas en acción, 2008, párr. 1).

Si se dirige la mirada hacia América Latina, se visibiliza que la tendencia hacia el consumo de ropa extranjera es un fenómeno innegable; muchas veces se adquieren prendas innecesarias, en las que los compradores se dejan llevar por el hecho de que utilizar tal marca, original o copia, proporciona cierto estatus que lo diferencia positivamente de otros; finalmente, lo importante es estar a la moda. En Ecuador, el fenómeno moda no se visibiliza como en los países europeos y norteamericanos; sin embargo, los ecuatorianos sí se ven influenciados por tendencias tardías que se incrustan en su entorno y que los hacen partícipes de un consumo irresponsable. Pocos son los usuarios que cuestionan y exigen la procedencia de los productos y realizan un consumo responsable.

\section{IMPACTOS MEDIOAMBIENTALES DE LA MODA}

A continuación, se presentan porcentajes reales de los impactos causados que la producción de la moda causa al medioambiente: 


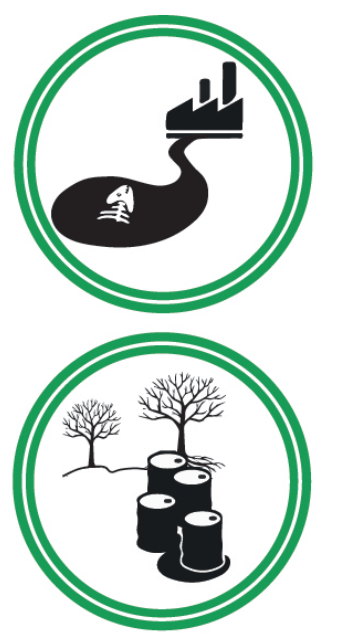

\section{PRODUCTOS QUÍMICOS}

La industria textil contamina un $20 \%$ de la aguas de toda la producción global. El uso intensivo de los productos químicos en los procesos de extracción y cultivo de materias primas y en los procesos de producción tienen un gran impacto en el medio ambiente, especialmente en el entorno acuático (ríos y mares).

\section{ESCASEZ DE AGUA}

El ciclo de vida de una camiseta de algodón requiere 2.700 litros de agua. La industria mundial utiliza 387.000 millones de litros de agua al año. Algunos productos textiles y algunas fases de la cadena de valor requieren el uso de gran cantidad de agua, con la consiguiente escasez para la vida humana y otros seres vivos.

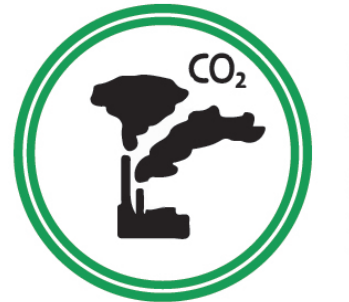

\section{GASES DE EFECTO INVERNADERO (GEI)}

La industria textil es la responsable del $10 \%$ del total de emisiones de $\mathrm{CO} 2$ en el mundo, con un consumo anual de un billón de kilovatios/hora.

La cantidad y la tipología de energía utilizada y de las emisiones generadas (CO2 y otros GEI) en la producción, el transporte, el uso y el mantenimiento de las prendas es un factor importante de la huella ecológica que deja el sistema moda.

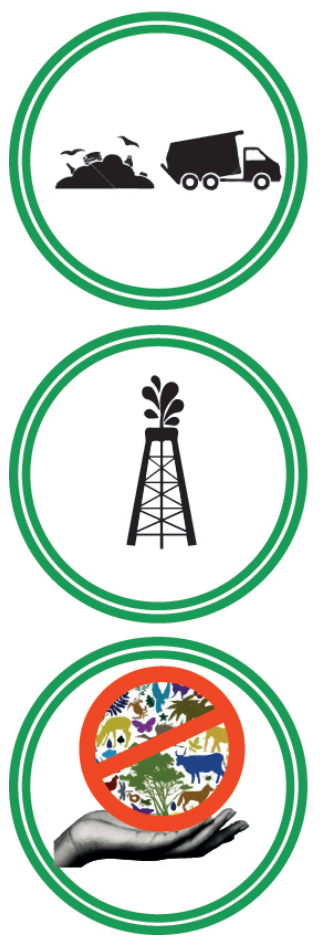

\section{RESIDUOS SÓLIDOS}

Los residuos de la industria textil suponen el $5 \%$ de los residuos totales (datos Estados Unidos).

A lo largo de la cadena de valor se generan muchos residuos. Los más importantes son las prendas que acaban en el vertedero, pero también lo son todos los embalajes no reutilizables o no reciclables que se desechan a lo largo de la cadena de valor.

\section{RECURSOS: TIERRA Y ENERGÍA}

El $58 \%$ de las fibras textiles producidas en el mundo derivan del petróleo. La fabricación de materias primas y, por extensión, la producción de materiales y productos acabados, por un lado, dependen de recursos finitos $y$, por otro lado, requieren cada vez más un uso intensivo de la tierra, que limita la dedicación a otros cultivos, como los alimentos.

\section{BIODIVERSIDAD}

En la India se han perdido las semillas de algodón debido a su contaminación por el algodón con transgénicos (Bt. México), cuna del maíz, ha perdido el $80 \%$ de sus variedades.

Figura 1. Cuadro referencial tomado de Salcedo, E. ,2014. Moda ética para un futuro sostenible, p. 28-29 (Autoría propia, 2015). 
En la búsqueda de la eficacia y eficiencia en los procesos y la generación de economías de escala, el ser humano está apostando por el concepto de monocultura, no solo en lo referente al diseño 0 a los procesos de manufactura, sino también en los tejidos y fibras y, por lo tanto, los cultivos y las especies. Este impacto no es fácil de medir, pero es importante reconocer una pérdida de biodiversidad.

Los daños que la producción textilera industrial causa afectan directamente al planeta que habitamos y, aunque los ecosistemas poseen cierta estabilidad y capacidad de amortiguación, a pesar de la explotación sostenida y superados determinados niveles de alteración, se producen cambios irreversibles que, en algunos casos, ponen en peligro la vida sobre el planeta. No se trata de lejanas fantasías alarmistas, sino de situaciones relativamente cercanas. Se puede constatar la situación visitando, por ejemplo, el Observatorio Meteorológico de Izaña y el Instituto Nacional de Meteorología, donde se trabaja en un proyecto de colaboración internacional, encaminado a medir la contaminación de diversos elementos como el anhídrido carbónico. Los datos obtenidos hasta el momento confirman que el nivel de CO2 en la atmósfera ha aumentado a razón de casi una parte por millón cada año. De mantenerse esa tendencia, en menos de quinientos años podría producirse un aumento medio de la temperatura de la superficie de la Tierra entre cinco y diez grados centígrados según la latitud, lo cual acarrearía la fusión de la mayor parte del hielo emergente en los casquetes polares y la consiguiente elevación del nivel de los océanos en varios metros; desaparecerían así algunas ciudades costeras, pues se inundarían.

Además de esta preocupación, podrían añadirse muchas otras de similar trascendencia, tales como:
[Los] procesos de edafogénesis que permiten la utilización continuada del suelo a salvo de los fenómenos de erosión, a la dinámica atmosférica que garantiza el mantenimiento de la calidad del aire, al correcto funcionamiento de los mecanismos de autodepuración de las aguas marinas y continentales y a las cadenas tróficas que sustentan la diversidad de los seres vivos (Nicolas, 1990, p. 3).

Después de hacer un análisis general de los impactos de la moda, es importante analizar los diferentes procesos de las fases del ciclo de vida de la prenda, para luego plantear soluciones viables en cada uno de estos planos. A continuación, se muestra un cuadro que detalla esta problemática: 

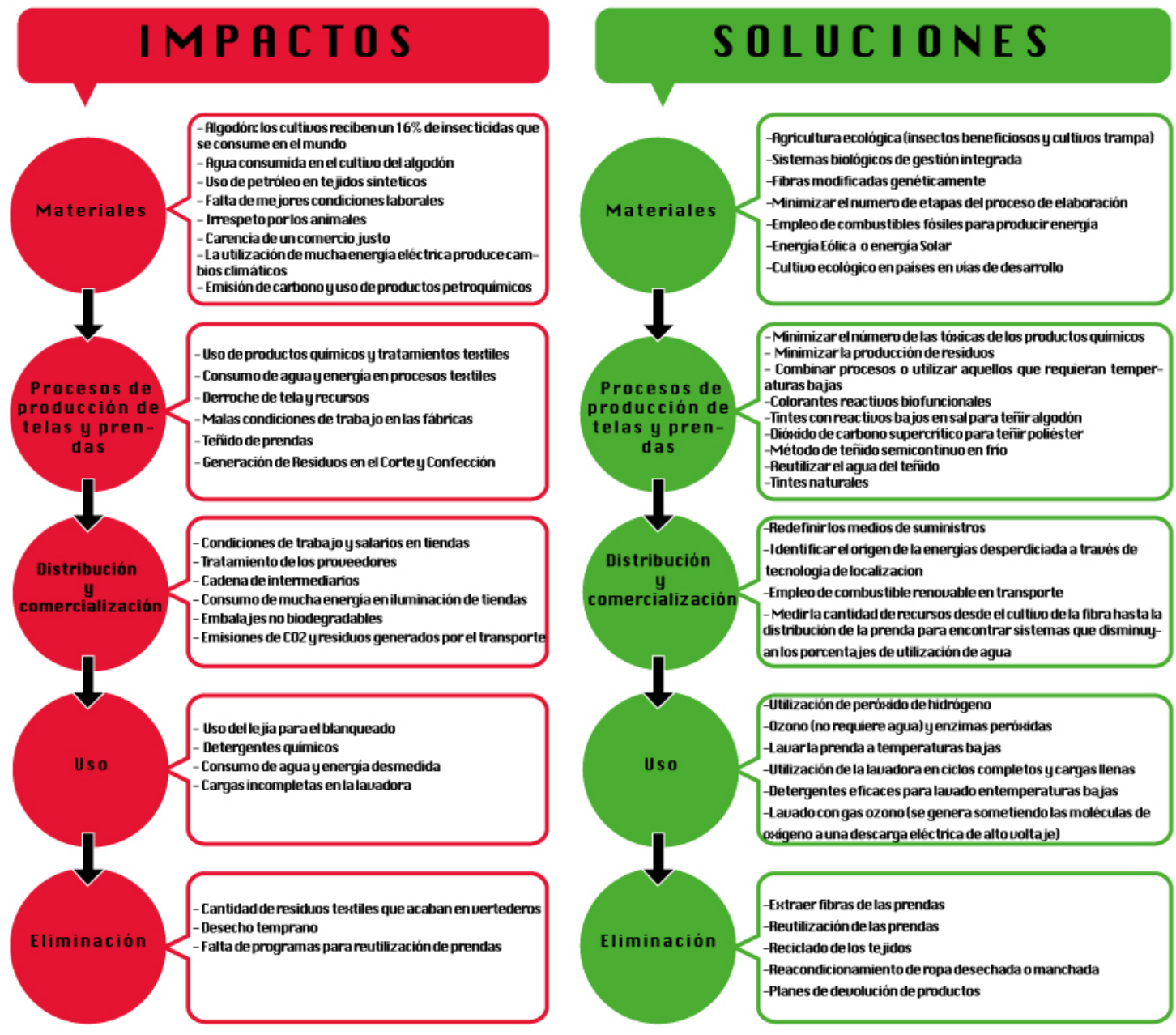

Figura 2. Datos tomados de Gwilt, Alison, 2014. Moda Sostenible, p. 14-45 y Grose Linda y Fletcher Kate, 2012. Gestionar la Sostenibilidad en la Moda, p. 13-73 (Autoría propia, 2015).

Son varias las alternativas que nos brinda la sostenibilidad, pero es importante realizar estudios minuciosos que puedan determinar los problemas y dónde se puede intervenir; es innegable que se puedan obviar varios de los procesos, pero sí es posible encontrar vías ejecutables en el camino ecológico y sustentable.

La idea de que la sostenibilidad augura un mundo mejor es cierta, en la medida en que propone una menor cantidad de residuos, contaminación, daños, destrucción, suelos esquilmados, trabajadores intoxicados, reducción de las masas de agua, y está basada en la reciprocidad, la complejidad y en profundo conocimiento de las pautas, la redes, los equilibrios y los ciclos que intervienen en el sistema de la moda (Grose \& Fletcher, 2012, p.11). 
Cada vez son más las empresas que se suman y se están adaptando con rapidez y agilidad a un modelo de producción ecológico, bajo una concepción de sostenibilidad y cuidado del medio ambiente y que adecúan sus procesos productivos a parámetros como valores de ética, responsabilidad social, utilización de recursos renovables y respeto al medio ambiente. Sin embargo, en el campo industrial algunas continúan produciendo bajo criterios del modelo tradicional: consumo, producción y marketing.

A diferencia de empresas que todavía contaminan sin medida, existen campañas colectivas dirigidas a conseguir que dichas empresas se comprometan a cumplir estas nuevas metas. Uno de esos casos es el de DETOX, dirigida por el grupo GREENPEACE, que, a través de sus gráficas, busca la concientización sobre la importancia de la conservación del planeta y de crear moda sin contaminación.
Se dice que puedes saber la ultimísima tendencia de la temporada mirando el color de los ríos de México y China. Esto ocurre porque las marcas mundiales de moda como Calvin Klein, GAP y Victoria's Secret están utilizando sustancias químicas peligrosas y colorantes para hacer nuestra ropa. Estas sustancias envenenan nuestros ríos, y rastros de estos productos químicos peligrosos también terminan permaneciendo en muchas de las prendas que compramos. Pero, ¡no tiene por qué ser así! [...] En todo el mundo, cientos de miles de personas están pidiendo a las marcas que la moda no tenga un coste para el planeta. Esta misma semana, el mayor fabricante del mundo, ZARA, se ha comprometido a limpiar su cadena de suministros, después de que más de 320.000 personas se unieran a la campaña, pidiendo a la marca una moda libre de tóxicos (Greenpeace, 2011, párr. 1).

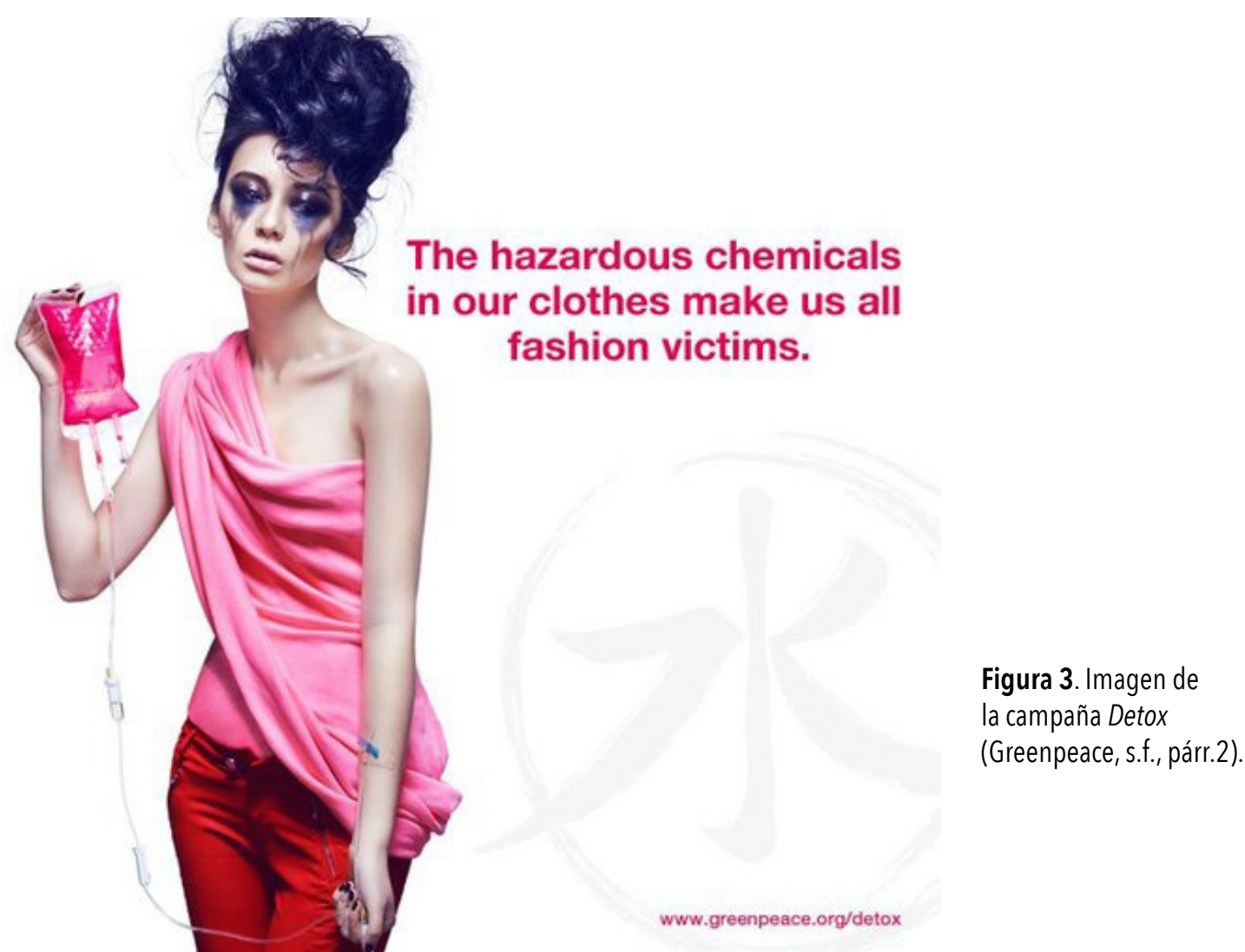




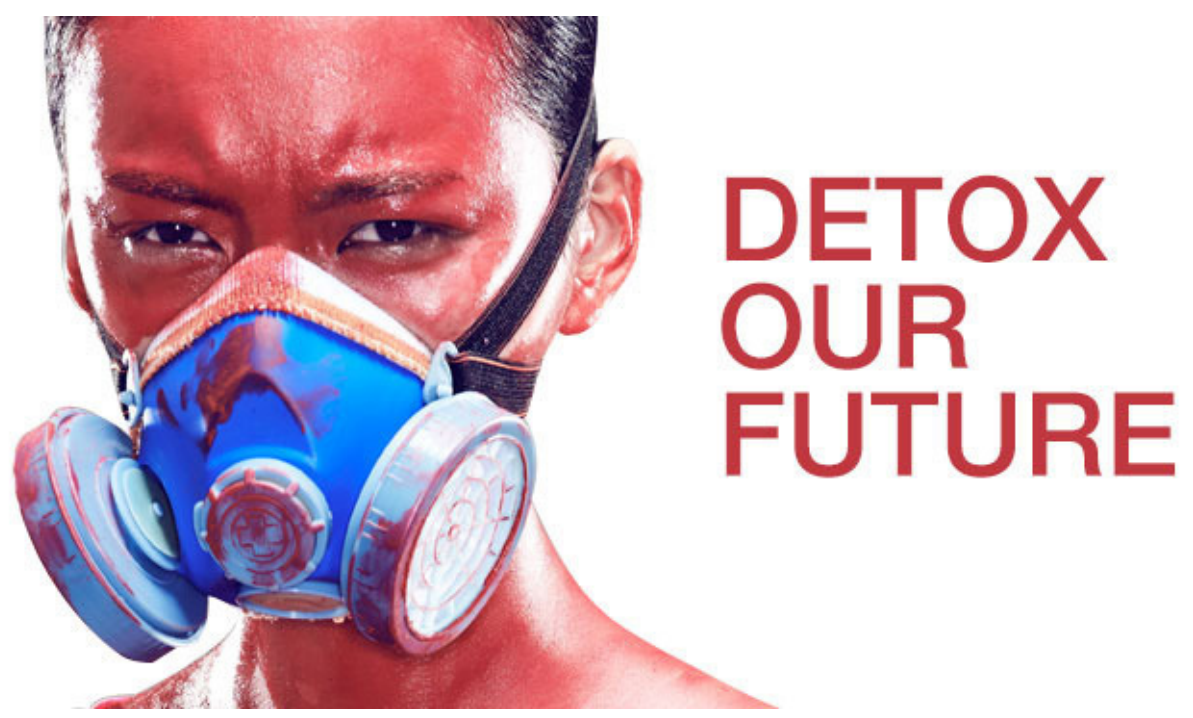

Figura 4. Imagen de la campaña Detox (Greenpeace, s.f., párr.1).

El proceso de sostenibilidad obliga a cambiar al sector de la moda. Cambiar hacia algo menos contaminante, más eficiente y más respetuoso de lo que existe hoy en día; cambiar la escala y la velocidad de estructuras en las que se sustenta y conectarlas entre sí. Como ejemplo de aplicación de este nuevo modelo sustentable y ecológico en el Ecuador, podríamos señalar el caso de la Empresa Enkador.

\section{ENKADOR. EMPRESA ECUATORIANA CON UN MODELO SOSTENIBLE EN SU PRODUCCIÓN}

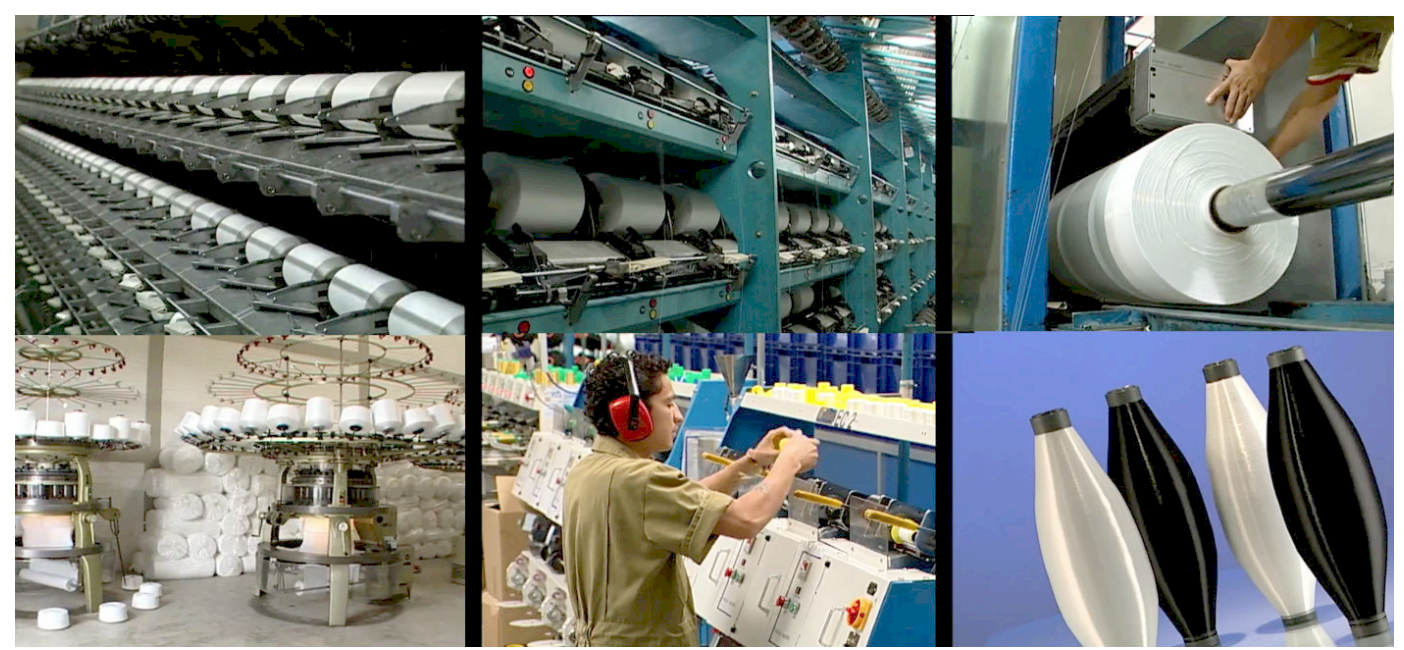

Figura 5. Imagen del proceso de botellas PET para la obtención de hilo (Enkador, 2013). 
Enkador es una empresa ecuatoriana que para dar solución a la problemática de la baja conciencia que tiene la población sobre el impacto ambiental que produce la basura, invirtió en el reciclado de botellas para obtener nuevos productos, ya sea envases, hilos de poliéster para fabricar prendas de vestir y alfombras, reIlenos de almohadas, entre otros; al tiempo que genera nuevas fuentes de empleo para más de mil personas.

Las botellas son elaboradas con plástico PET (Polietiléntereftalato), y su consumo en el mundo supera los 19 millones de toneladas anuales, cifras del 2013, según datos publicados en el video institucional. Estos productos se constituyen en parte importante de la basura que se genera anualmente, $45 \%$ en volumen, más si consideramos que actualmente solo se recicla el $20 \%$ de dicho volumen. Se utilizan esencialmente como envases para bebidas carbonatadas, aguas, aceite, alimentos, medicamentos y cosméticos. Tras cumplir su función estas botellas terminan su ciclo de vida como recipientes. Sin embargo, si luego se convierten en materia prima de nuevos productos se inicia así un nuevo ciclo.

Esta ha sido una de las propuestas ejecutadas por Enkador en Ecuador: usa las botellas para fabricar hilos de filamento de poliéster y poliamida. La fábrica mantiene una producción promedio de $6.000 \mathrm{Kg}$ al año, de los cuales el $47 \%$ se exporta a Colombia, Venezuela, Chile, Perú y Centroamérica y el 53\% restante se comercializa dentro del país, solventa las necesidades del mercado nacional. Los productos fabricados por la empresa se utilizan como materia prima para la producción de tejidos planos y tubulares, etiquetas, cintas, cordones y para la confección de prendas de vestir, calzado y artículos de cuero. Desde abril del 2004, la empresa obtuvo la certificación de la norma internacional ISO 9001-2002, lo que garantiza la calidad de todos los artículos que produce (Saldarriaga, 2010).

\section{PROPUESTA DE DISEÑO ECOLÓGICOY SUSTENTABLE EN ECUADOR}

Al reconocer los escenarios actuales, es pertinente pensar en propuestas que se podrían aplicar en el Ecuador para crear de ese modo una vinculación entre la academia y el diseño $y$, a su vez, que permitan la intervención de las empresas públicas y privadas con el fin de instaurar una conciencia ecológica y un consumo responsable en la sociedad. Para ello, tomamos como referencia lo establecido por Offray Vladimir Luna Cárdenas (2014) en su ensayo Ecología de Saberes en Diseño cuando señala que:

los saberes en diseño son buenos candidatos a ser considerados sistemas complejos: son diversos, no lineales, interconectados y dinámicos. Si partimos de la hipótesis de que tales saberes conforman efectivamente sistemas complejos interconectados entre sí, los análisis ecológicos desde sistemas complejos pueden ser una buena inspiración sobre cómo mapear y representar las conexiones actuales y posibles de los saberes en diseño entre sí y dar cuenta de cómo ellos conforman una ecología de saberes. Esta hipótesis de partida tomará más fuerza en la medida en que desarrollemos la propuesta que ella nos permite. Si se quiere, esto puede ser un tipo de pensamiento circular, pero no uno tautológico, sino autopoiético, que emplea un proceso de boots- 
traping sencillo, la de que los saberes en diseño constituyen una red compleja para jalonar estados más avanzados de sí mismos, la de que la sociedad es una red compleja autopoiética y esto tiene consecuencias en las epistemologías y acciones del diseño (Luna, 2014, p.3).

Con estos nuevos pensamientos que rompen los paradigmas establecidos, en donde las disciplinas actúan individualmente, se abren nuevas oportunidades para los diseñadores, para que creen proyectos y programas multidisciplinarios e interdisciplinarios, en donde se tengan nuevas miradas para establecer soluciones innovadoras bajo un modelo sustentable y ecológico a las problemáticas que se presenten. Es por esto que una propuesta de solución a una problemática de nuestro medio se considera pertinente e importante, con el fin de mitigar el impacto de la industria textil y los residuos que se generan en la producción.

En el modelo tradicional son pocas las vinculaciones entre industria y academia; si bien se ejecutan proyectos de corto plazo, estos se desvanecen con el tiempo y pierden su proyección inicial. En un gráfico se podría ilustrar de esta manera:

Una propuesta consiste en vincular a

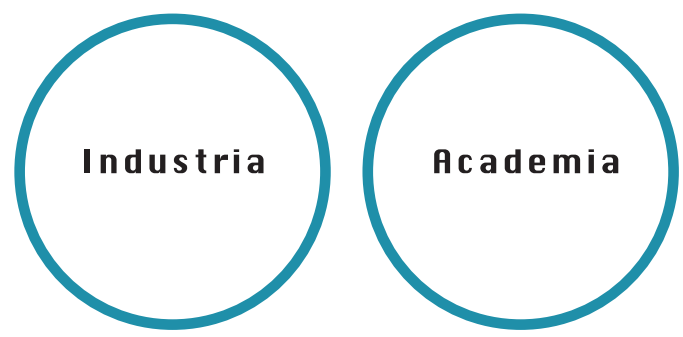

Figura 6. Industria y academia (Autoría propia, 2015). las dos mediante el diseño, de tal manera que la academia sea capaz de dar soluciones a los diferentes problemas que se presentan en mundo real. Ciertamente, el diseño es una disciplina que puede trabajar en programas interdisciplinarios que ayudan a establecer nuevas miradas y soluciones, crear caminos sustentables con el medio ambiente.

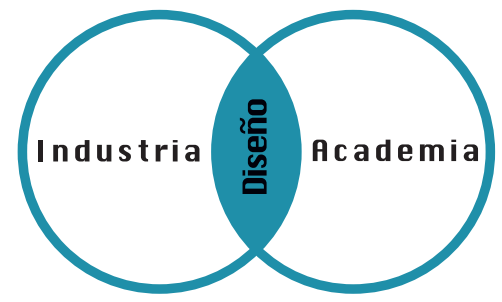

Figura 7. Industria, academia y diseño (Autoría propia, 2015).

Por ejemplo, ha sido evidente que una problemática latente en el Ecuador es la gran cantidad de excedentes de retazos de telas generados por las industrias textiles nacionales. En este contexto, la academia puede intervenir de las siguientes maneras:

- Realizar proyectos con los alumnos de carreras de diseño de indumentaria y diseño de productos y plantear propuestas de reutilización de estos retazos para que puedan ser aplicados en nuevas prendas u objetos textiles.

- Crear proyectos multidisciplinarias, por ejemplo, con la carrera de Ingeniería Civil, para utilizar estos excedentes como relleno de fundiciones.

- Incorporar máquinas desfriladoras en 
su infraestructura, que permitan crear nuevas fibras a partir de los retazos.

- Realizar convenios con las facultades de diseño del país para intervenir en la capacitación de las empresas, en sistemas CAD / CAM que disminuyan los residuos en el corte.

- Proponer talleres interactivos, en los que participen niños y personas de la tercera edad para elaborar manualidades y potenciar su capacidad de motricidad.

\section{REFLEXIONES FINALES}

Se considera que una transformación de nuestra forma de pensar y la generación de un compromiso ante la situación del medio ambiente es necesaria en todas las partes involucradas para que así se realicen esfuerzos de una forma integral y ética. Dicha transformación depende del conocimiento, de los medios y las publicaciones que apelan al sentido innato del ser humano de proteger y cuidar la vida.

Es innegable que la situación actual de nuestro planeta en relación al medioambiente debe ser una preocupación de todos. Las decisiones que se tomen hoy, tendrán sus repercusiones el día de mañana.

Por tanto, es responsabilidad de cada uno de nosotros desarrollar acciones conscientes para utilizar los recursos naturales, sin causar daños colaterales a nuestro entorno. En el camino de la sostenibilidad y la ecología se abren nuevas oportunidades para los diseñadores en programas multidisciplinarios que resulten en mejores condiciones de vida para todos. 


\section{REFERENCIAS BIBLIOGRÁFICAS}

Greenpeace. (2011), Tóxicos fuera de temporada. Estados Unidos. Recuperado de: http://www.greenpeace.org/espana/es/Trabajamos-en/Parar-la-contaminacion/Agua/Campana-Detox-/Fashion-Detox-1

Grose, L. y Fletcher, K. (2012). Gestionar la sostenibilidad en la Mmda. Barcelona: Blume.

Gwilt, A. (2014). Moda Sostenible. Barcelona: Gustavo Gili.

Luna, 0. (2014). Ecología de Saberes en Diseño. Un ejemplo desde los discursos autopoiéticos. Recuperado de: " http://mutabit.com/deltas/repos. fossil/doctorado-offray/doc/tip/Tesis/ExamenCandidatura/Escrito2/luna-offray-ecologia-de-saberes-en-diseno.pdf

Nicolas, J. (1990). Ecología y gestión ecológica. Entre la estática y la subsistencia. La Laguna: Universidad de La Laguna.

Salcedo, E. (2014). Moda ética para un futuro sostenible. Barcelona: Gustavo Gili.

Saldarriaga, C. (2010). Estudio de impacto ambiental Expost o auditoría ambiental inicial. Quito: Proyecto Enkador.

\section{FIGURAS}

Figura 1. Salcedo, E. (2014). Moda ética para un futuro sostenible, p. 28-29, Barcelona: Gustavo Gili.

Figura 2. Gwilt, A. (2014). Grose, L. Fletcher, K. (2012). Moda Sostenible, p. 1445 y Gestionar la Sostenibilidad en la Moda, p. 13-73. Barcelona: Blume.

Figura 3. Greenpeace. (2011). Exige a Zara que se una al reto Detox, Estados Unidos. Recuperado de: http://www.greenpeace.org/mexico/es/Campanas/ Toxicos/Contaminacion-de-nuestros-rios/Detox/Zara/

Figura 4. Greenpeace, (2011). Exige a Zara que se una al reto Detox, Estados Unidos. Recuperado de: http://www.greenpeace.org/international/en/ campaigns/detox/

Figura 5. Enkador, (2013). Documental Aprobado Enkador July 17, Ecuador. Recuperado de: https://www.youtube.com/watch?v=ezY_zKrWJ_4 
